\title{
High-pressure study of thermodynamic parameters of diamond-type structured crystals using interatomic Morse potentials
}

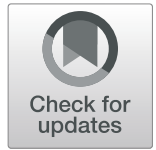

\author{
Duc Ba Nguyen ${ }^{*}$ (D) and Hiep Phi Trinh
}

\author{
*Correspondence: ducnb@ \\ daihoctantrao.edu.vn \\ Tan Trao University, Tuyen Quang, \\ Vietnam
}

\begin{abstract}
In this work, we have determined the mean square relative displacement, elastic constant, anharmonic effective potential, correlated function, local force constant, and other thermodynamic parameters of diamond-type structured crystals under high-pressure up to $14 \mathrm{GPa}$. The parameters are calculated through theoretical interatomic Morse potential parameters, by using the sublimation energy, the compressibility, and the lattice constant in the expanded X-ray absorption fine structure spectrum. Numerical results agree well with the experimental values and other theories.
\end{abstract}

Keywords: Morse potential parameter, State equation, Correlation function, Elastic constant, Mean square relative displacement

\section{Introduction}

High-pressure research is a very active research field. Recent progress has been recently made in characterizing elastic, mechanical, and other physical properties of material [1-3]. The use of interatomic Morse potentials in Expanded X-ray Absorption Fine Structure (EXAFS) theory to study thermodynamic parameters under highpressure currently also attracts the attention of materials scientists.

In EXAFS spectra with the anharmonic effects, the anharmonic Morse potential [4] is suitable for describing the interaction and oscillations of atoms in the crystals [5]. In the EXAFS theory, photoelectrons are emitted by the absorber scattered by surrounded vibrating atoms. This thermal oscillation of atoms contributes to the EXAFS spectra, especially the anharmonic EXAFS [6, 7], which is affected by these spectra's physical information. In the EXAFS spectrum analysis, the parameters of interatomic Morse potential are usually extracted from the experiment. Because experimental data are not available in many cases, a theory is necessary to deduce interatomic Morse potential parameters. The only calculation has been carried out for cubic crystals by using anharmonic correlated Einstein model [8]. The results have been used actively for calculating EXAFS thermodynamic parameters [9] and are reasonable with those extracted

(c) The Author(s). 2021 Open Access This article is licensed under a Creative Commons Attribution 4.0 International License, which permits use, sharing, adaptation, distribution and reproduction in any medium or format, as long as you give appropriate credit to the original author(s) and the source, provide a link to the Creative Commons licence, and indicate if changes were made. The images or other third party material in this article are included in the article's Creative Commons licence, unless indicated otherwise in a credit line to the material. If material is not included in the article's Creative Commons licence and your intended use is not permitted by statutory regulation or exceeds the permitted use, you will need to obtain permission directly from the copyright holder. To view a copy of this licence, visit http://creativecommons.org/licenses/by/4.0/. The Creative Commons Public Domain Dedication waiver (http://creativecommons.org/publicdomain/zero/1.0/) applies to the data made available in this article, unless otherwise stated in a credit line to the data. 
from EXAFS data [10]. Therefore, the requirement for calculation of the anharmonic interatomic Morse interaction potential due to thermal disorder for other structures is essential.

The purpose of this study is to expand a method to calculate the interatomic Morse potential parameters using the energy of sublimation, the compressibility, and the lattice constant with the effect of the disorder of temperature. The received interatomic Morse potential parameters are used to calculate the mean square relative displacement (MSRD), mean square displacement (MSD), elastic constant, anharmonic interatomic effective potential, and effective local force constant for diamond-type (DIA) structure crystals such as silicon ( $\mathrm{Si}$ ), germanium $(\mathrm{Ge})$, and SiGe semiconductor. Numerical results are in agreement with the experimental values and other theories [10-14].

Diamond's cubic structure is in the Fd3m space group, which follows the facecentered cubic Bravais lattice (Fig. 1). The lattice describes the repeat pattern, for diamond cubic crystals, the lattice of two tetrahedrally bonded atoms in each primitive cell, separated by $1 / 4$ of the width of the unit cell in each dimension. The diamond lattice can be viewed as a pair of intersecting face-centered cubic lattices, with each separated by $1 / 4$ of the width of the unit cell in each dimension. The atomic packing factor of the diamond cubic structure is $\pi \sqrt{ } 3 / 16$, significantly smaller (indicating a less dense structure) than the packing factors for the face-centered-cubic lattices. The first-, second-, third-, fourth-, and fifth-nearest-neighbor distances in units of the cubic lattice constant are $\sqrt{ } 3 / 4, \sqrt{ } 2 / 2, \sqrt{ } 11 / 4,1$, and $\sqrt{ } 19 / 4$, respectively.

\section{Methods}

- This study uses the theoretical method to calculate the parameter of interatomic Morse potential.

- Using the obtained interatomic Morse potential parameters to determine state equations, calculate some thermodynamic parameters that depend on temperature and pressure for some pure and doped crystals with a cubic structure.

- Compare the theoretical results with experimental data.

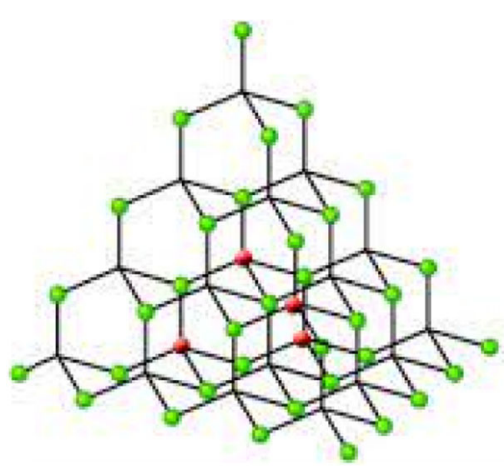

a)

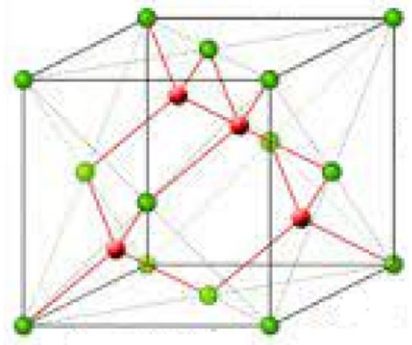

b)

Fig. 1 Style of diamond's cubic structure is in the Fd3m space group 


\section{Methodology}

The $\varepsilon\left(\mathrm{r}_{\mathrm{ij}}\right)$ potential of atoms $\mathrm{i}$ and $\mathrm{j}$ separated by a distance $\mathrm{r}_{\mathrm{ij}}$ is given in by the Morse function:

$$
\varepsilon\left(\mathrm{r}_{\mathrm{ik}}\right)=\mathrm{D}\left\{\mathrm{e}^{-2 \alpha\left(\mathrm{r}_{\mathrm{ij}}-\mathrm{r}_{\mathrm{o}}\right)}-2 \mathrm{e}^{-\alpha\left(\mathrm{r}_{\mathrm{ij}}-\mathrm{r}_{\mathrm{o}}\right)}\right\},
$$

where $1 / \alpha$ describes the width of the potential, $D$ is the dissociation energy $\left(\varepsilon\left(\mathrm{r}_{0}\right)=-\right.$ $D) ; r_{0}$ is the equilibrium distance of the two atoms.

To obtain the potential energy of a large crystal whose atoms are at rest, it is necessary to sum Eq. (1) over the entire crystal. It is quickly done by selecting an atom in the lattice as origin, calculating its interaction with all others in the crystal, and then multiplying by $N / 2$, where $N$ is the total number of atoms in a crystal. Therefore, the potential $E$ is given by:

$$
E=\frac{1}{2} N D \sum_{j}\left\{e^{-2 \alpha\left(r_{j}-r_{o}\right)}-2 e^{-\alpha\left(r_{j}-r_{o}\right)}\right\}
$$

Here $r_{\mathrm{j}}$ is the distance from the origin atom to the jth atom. It is beneficial to describe the following quantities:

$$
r_{j}=\left[m_{j}^{2}+n_{j}^{2}+l_{j}^{2}\right]^{1 / 2} a=M_{j} a
$$

where $m_{j}, n_{j}, l_{j}$ are position coordinates of atoms in the lattice. Substitute the Eq. (3) into Eq. (2), the potential energy can be rewritten as:

$$
E(a)=\frac{1}{2} N D e^{\alpha r_{0}}\left[e^{\alpha r_{0}} \sum_{j} e^{-2 \alpha a M_{j}}-2 \sum_{j} e^{-\alpha a M_{j}}\right] .
$$

The first and second derivatives of the potential energy of Eq. (4) concerning $a$, we have:

$$
\begin{aligned}
& \frac{d E}{d a}=-\alpha N D e^{\alpha r_{0}}\left[e^{\alpha r_{0}} \sum_{j} M_{j} e^{-2 \alpha a M_{j}}+\sum_{j} M_{j} e^{-\alpha a M_{j}}\right], \\
& \frac{d^{2} E}{d a^{2}}=\alpha^{2} N D e^{\alpha r_{0}}\left[2 e^{\alpha r_{0}} \sum_{j} M_{j}^{2} e^{-2 \alpha a M_{j}}-\sum_{j} M_{j}^{2} e^{-\alpha a M_{j}}\right] .
\end{aligned}
$$

At absolute zero $T=0, \mathrm{a}_{0}$ is the value of $a$ for which the lattice is in equilibrium, then $E\left(a_{0}\right)$ gives the energy of cohesion, $\left[\frac{d E}{d a}\right]_{a_{0}}=0$, and $\left[\frac{d^{2} E}{d a^{2}}\right]_{a_{0}}$ is related to the compressibility [15]. That is,

$$
d E\left(a_{0}\right)=E_{0}\left(a_{0}\right),
$$

where $E_{0}\left(a_{0}\right)$ is the energy of sublimation at zero pressure and temperature,

$$
\left(\frac{d E}{d a}\right)_{a_{0}}=0
$$

and the compressibility is given by [8] 


$$
\frac{1}{\mathrm{\kappa}_{0}}=V_{0}\left(\frac{d^{2} E_{0}}{d V^{2}}\right)_{a_{0}}=V_{0}\left(\frac{d^{2} E}{d V^{2}}\right)_{a_{0}},
$$

where $V_{0}$ is the volume at $T=0$ and $\mathrm{k}_{0}$ is compressibility at zero temperature and pressure. The volume per atom $V / N$ is related to the lattice constant $a$ by

$$
\frac{V}{N}=c a^{3}
$$

Substituting Eq. (10) into Eq. (9) the compressibility is formulated by

$$
\frac{1}{\mathrm{\kappa}_{0}}=\frac{1}{9 c N a_{0}}\left(\frac{d^{2} E}{d a^{2}}\right)_{a=a_{0}} .
$$

Using Eq. (5) to solve Eq. (8), we obtain

$$
e^{\alpha r_{0}}=\frac{\sum_{j} M_{j} e^{-\alpha a M_{j}}}{\sum_{j} M_{j} e^{-2 \alpha a M_{j}}} .
$$

From Eqs. $(4,6,7,11)$, we derive the relation

$$
\frac{e^{\alpha r_{0}} \sum_{j} e^{-2 \alpha a M_{j}}-2 \sum_{j} e^{-\alpha a M_{j}}}{4 \alpha^{2} e^{\alpha r_{0}} \sum_{j} M_{j}^{2} e^{-2 \alpha a M_{j}}-2 \alpha^{2} \sum_{j} M_{j}^{2} e^{-\alpha a M_{j}}}=\frac{E_{0} \kappa_{0}}{9 c N a_{0}} .
$$

Solving the system of Eq. $(12,13)$, we obtain $\alpha$ and $r_{0}$. Using $\alpha$ and Eq. (4) to solve Eq. (7), we have $D$. The interatomic Morse potential parameters $D, \alpha$ depend on the compressibility $\kappa_{0}$, the energy of sublimation $E_{0}$, and the lattice constant $a$. These values of all crystals are available already [16].

Next, we apply the above expressions to claculate the equation of state and elastic constants. It is possible to calculate the state equation from the potential energy $E$. If we assumed that the Debye model could express the thermal section of the free energy, then the Helmholtz energy is given by [8]

$$
\begin{aligned}
& F=E+3 N k_{B} T \ln \left(1-e^{-\theta_{D} / T}\right)-N k_{B} T D\left(\theta_{D} / T\right) \\
& D\left(\frac{\theta_{D}}{T}\right)=3\left(\frac{T}{\theta_{D}}\right)^{3} \int_{0}^{\theta_{D} / T} \frac{x^{3}}{e^{x}-1} d x
\end{aligned}
$$

where $k_{B}$ is Boltzmann constant, and $\theta_{\mathrm{D}}$ is Debye temperature.

Using Eqs. $(14,15)$, we derive the equation of state as

$$
P=-\left(\frac{\partial F}{\partial V}\right)_{T}=\frac{1}{3 c a^{2}} \frac{d E}{d a}+\frac{3 \gamma_{G} R T}{V} D\left(\frac{\theta_{D}}{T}\right),
$$

where $\gamma_{G}$ is the Grüneisen parameter, and $V$ is the volume.

After transformations, the Eq. (16) is resulted as 


$$
\begin{aligned}
& P=\frac{\left[N D e^{\alpha r_{0}} \alpha \sum_{j} M_{j} e^{-\alpha a_{0} M_{j}(1-x)^{1 / 3}}\right]}{3 c a_{0}^{2}(1-x)^{2 / 3}}-N D e^{2 \alpha r_{0}} \alpha \sum_{j} M_{j} e^{-2 \alpha a_{0} M_{j}(1-x)^{1 / 3}}+\frac{3 \gamma_{G} R T}{V_{0}(1-x)} D\left(\frac{\theta_{D}}{T}\right), \\
& x=\frac{V_{0}-V}{V_{0}}, \quad V_{0}=c a_{0}^{3}, \quad R=N k_{B}, \quad N=6.02 \times 10^{23} .
\end{aligned}
$$

The equation of state (17) contains the obtained interatomic Morse potential parameters; $c$ is a constant and has value according to the structure of the crystal.

An elastic tensor describes the elastic properties of a crystal in the crystal's motion equation. The non-vanishing components of the elastic tensor are defined as elastic constants. They are given for crystals of lattice structure by [17]:

$$
\begin{aligned}
& c_{11}=c_{22}=\sqrt{2} r_{0}\left[10 \Psi^{\prime \prime}\left(r_{0}^{2}\right)+16 \Psi^{\prime \prime}\left(2 r_{0}^{2}\right)+81 \Psi^{\prime \prime}\left(3 r_{0}^{2}\right) \cdots\right]-\frac{\left\{\sqrt{\frac{2}{3}}\left[-2 \Psi^{\prime \prime}\left(r_{0}^{2}\right)+16 \Psi^{\prime \prime}\left(2 r_{0}^{2}\right)-40 \Psi^{\prime \prime}\left(3 r_{0}^{2}\right) \cdots\right]\right\}^{2}}{\sqrt{2} r_{0}^{-1}\left[4 \Psi^{\prime \prime}\left(r_{0}^{2}\right)+16 \Psi^{\prime \prime}\left(2 r_{0}^{2}\right)+12 r_{0}^{-1} \Psi^{\prime}\left(2 r_{0}^{2}\right) \cdots\right]} \\
& c_{12}=\frac{\sqrt{2} r_{0}\left[10 \Psi^{\prime \prime}\left(r_{0}^{2}\right)+16 \Psi^{\prime \prime}\left(2 r_{0}^{2}\right)+81 \Psi^{\prime \prime}\left(3 r_{0}^{2}\right) \cdots\right]}{3}+\frac{\left\{\sqrt{\frac{2}{3}}\left[-2 \Psi^{\prime \prime}\left(r_{0}^{2}\right)+16 \Psi^{\prime \prime}\left(2 r_{0}^{2}\right)-40 \Psi^{\prime \prime}\left(3 r_{0}^{2}\right) \cdots\right]\right\}^{2}}{\sqrt{2} r_{0}^{-1}\left[4 \Psi^{\prime \prime}\left(r_{0}^{2}\right)+16 \Psi^{\prime \prime}\left(2 r_{0}^{2}\right)+12 r_{0}^{-1} \Psi^{\prime}\left(2 r_{0}^{2}\right) \cdots\right]}, \\
& c_{33}=\frac{\sqrt{2}}{3} r_{0}\left[32 \Psi^{\prime \prime}\left(r_{0}^{2}\right)+32 \Psi^{\prime \prime}\left(2 r_{0}^{2}\right)+\frac{512}{3} \Psi^{\prime \prime}\left(3 r_{0}^{2}\right)+\cdots\right] \\
& c_{13}=c_{23}=\sqrt{2} r_{0}\left[8 \Psi^{\prime \prime}\left(r_{0}^{2}\right)+32 \Psi^{\prime \prime}\left(2 r_{0}^{2}\right)+112 \Psi^{\prime \prime}\left(3 r_{0}^{2}\right)+\cdots\right] \\
& \Psi^{\prime}(r)=-2 D \alpha\left[e^{-2 \alpha\left(r-r_{0}\right)}-e^{-\alpha\left(r-r_{0}\right)}\right] \frac{1}{r}, \\
& \Psi^{\prime \prime}(r)=D \alpha^{2}\left[2 e^{-2 \alpha\left(r-r_{0}\right)}-\frac{1}{2} e^{-\alpha\left(r-r_{0}\right)}\right] \frac{1}{r^{2}}+D \alpha\left[e^{-2 \alpha\left(r-r_{0}\right)}-e^{-\alpha\left(r-r_{0}\right)}\right] \frac{1}{2 r^{3}} .
\end{aligned}
$$

Hence, the derived elastic constants contain the interatomic Morse potential parameters.

Next, apply to calculate of anharmonic interatomic effective potential and local force constant in EXAFS theory. The expression for the anharmonic EXAFS function [2] is described by

$$
\chi(k)=A(k) \frac{\exp [-2 \Re / \lambda(k)]}{k \Re^{2}} \operatorname{Im}\left\{e^{i \phi(k)} \exp \left[2 i k \Re+\sum_{n} \frac{(2 i k)^{n}}{n !} \sigma^{(n)}\right]\right\},
$$

where $A(k)$ is scattering amplitude of atoms, $\phi(K)$ is the total phase shift of photoelectron, and $k$ and $\lambda$ are wave number and mean free path of the photoelectron, respectively. The $\sigma^{(\mathrm{n})}$ are the cumulants; they describe asymmetric of anharmonic interatomic Morse potential, due to the average of the function $e^{-2 \mathrm{ikr}}, \mathfrak{R}=\langle r\rangle$, and $r$ is the instantaneous bond length between absorber and backscatter atoms at $T$ temperature.

For describing anharmonic EXAFS, effective anharmonic potential [9] of the system is derived which in the current theory is expanded up to the third order and given by

$$
\mathrm{E}_{\text {eff }}(\mathrm{x})=\frac{1}{2} \mathrm{k}_{\text {eff }} \mathrm{x}^{2}+\mathrm{k}_{3 \text { eff }} \mathrm{x}^{3}+\ldots+=\mathrm{E}(\mathrm{x})+\sum_{\mathrm{j} i \mathrm{i}} \mathrm{E}\left(\frac{\mu}{M_{\mathrm{i}}} \mathrm{xR}_{12}, \hat{\mathrm{R}}_{\mathrm{ij}}\right), \quad \mu=\frac{M_{1} M_{2}}{M_{1}+M_{2}} ; \quad \hat{R}=\frac{\mathfrak{R}}{|R|} .
$$

Here, $k_{\text {eff }}$ is the effective local force constant, and $k_{3 \text { eff }}$ is the cubic parameter characterizing the asymmetry in the pair interatomic Morse potential, and $x$ is the deviation of instantaneous bond length between the two atoms from equilibrium. The correlated 
model defined as the oscillation of a pair of particles with $M_{1}$ and $M_{2}$ mass. Their vibration influenced by their neighbors atoms given by the sum in Eq. (24), where the sum $\mathrm{i}$ is over absorber $(i=1)$ and backscatterer $(i=2)$, and the sum $j$ is over all their near neighbors, excluding the absorber and backscatterer themselves whose contributions are described by the term $\mathrm{E}(\mathrm{x})$. The advantage of this model is a calculation based on including the contributions of the nearest neighbors of absorber and backscatter atoms in EXAFS. The anharmonic interatomic effective potential Eq. (26) has the form

$$
E_{e f f}(x)=E_{x}(x)+2 E_{x}\left(-\frac{x}{2}\right)+8 E_{x}\left(-\frac{x}{4}\right)+8 E_{x}\left(\frac{x}{4}\right) .
$$

Applying interatomic Morse potential given by Eq. (1) expanded up to 4th order around its minimum point

$$
E_{e f f}(x)=D\left(e^{-2 \alpha x}-2 e^{-\alpha x}\right) \approx D\left(-1+\alpha^{2} x^{2}-\alpha^{3} x^{3}+\frac{7}{12} \alpha^{4} x^{4} \ldots\right) .
$$

From Eqs. (26)-(28), we obtain the anharmonic effective potential $E_{\text {eff }}$, effective local force constant $k_{\text {eff }}$, anharmonic parameters $k_{3 \text { eff }}$ for lattice crystals presented in terms of our calculated interatomic Morse potential parameters $D$ and $\alpha$.

In Eq. (25), $\sigma^{(\mathrm{n})}$ is cumulants, in which $\sigma^{2}(T)$ is the Debye-Waller factor (DWF) or MSRD [9]. In the diffraction or X-ray absorption, the DWF has a form similar $u^{2}(T)$. In the EXAFS spectrum, DWF is regarded as to correlated averages over the relative displacement of $\sigma^{2}(T)$ for a pair of atoms, while neutron diffraction allude to the MSD $u^{2}(T)$ of an atom [18]. From $\sigma^{2}(T)$ and $u^{2}(T)$, the correlated function $C_{\mathrm{R}}(T)$ to describe the effects of correlation in the vibration of atoms can be deduced. Using the anharmonic correlated Debye model (ACDM), the MSRD $\sigma^{2}(T)$ has the form [19]:

$$
\begin{aligned}
& \sigma^{2}(T)=\frac{\hbar a}{10 \pi \mathrm{D} \alpha^{2}} \int_{0}^{\pi / a} \omega_{A}(q) \frac{1+z_{A}(q)}{1-z_{A}(q)} d q, \\
& z(q)=e^{-\left(\beta \hbar \omega_{A}(q)\right)}, \quad \omega_{A}(q)=2 \sqrt{\frac{10 \mathrm{D} \alpha^{2}}{M}}|\sin (q a / 2)|, \quad|q| \leq \pi / \mathrm{a} .
\end{aligned}
$$

Similarly, for the anharmonic Debye model, $u^{2}(T)$ have been determined as:

$$
u^{2}(T)=\frac{\hbar a}{16 \pi \mathrm{D} \alpha^{2}} \int_{0}^{\pi / a} \omega_{D}(q) \frac{1+z_{D}(q)}{1-z_{D}(q)} d q
$$

Table 1 Morse potential parameters $D, a$ and the related parameter $r_{0}$ of Si, Ge, and SiGe in comparison to some experimental results $[10,14]$

\begin{tabular}{lllll}
\hline Crystal & $\boldsymbol{\beta}$ & $\boldsymbol{a}\left(\AA^{-\mathbf{1}}\right)$ & $\boldsymbol{D}(\mathbf{e V})$ & $\boldsymbol{r}_{\mathbf{0}}(\AA)$ \\
\hline Si (present) & 120.110 & 1.3642 & 0.9862 & 2.8429 \\
Si (expt.) & - & 1.3106 & - & 2.7503 \\
Ge (present) & 327.210 & 1.5569 & 0.9675 & 2.8319 \\
Ge (expt.) & - & 1.4105 & - & 2.7442 \\
SiGe (present) & - & 1.4606 & 0.9769 & 2.7934 \\
\hline
\end{tabular}


Table 2 Values elastic constants $\left(\times 10^{-11} \mathrm{~N} / \mathrm{m}\right)$ for $\mathrm{Si}$, Ge by present theory and experimental values [11]

\begin{tabular}{lllll}
\hline Crystal & $\boldsymbol{c}_{\mathbf{1 1}}$ & $\boldsymbol{c}_{\mathbf{1 2}}$ & $\boldsymbol{c}_{\mathbf{1 3}}$ & $\boldsymbol{c}_{\mathbf{3 3}}$ \\
\hline Si (present) & 1.85 & 0.64 & 0.55 & 2.13 \\
Si (expt.) & 1.77 & 0.41 & 0.61 & 1.54 \\
Ge (present) & 1.46 & 0.57 & 0.46 & 1.63 \\
Ge (expt.) & 1.35 & 0.52 & 0.52 & 0.57 \\
\hline
\end{tabular}

$$
z_{D}(q)=e^{-\left(\beta \hbar \omega_{D}(q)\right)}, \quad \omega_{D}(q)=2 \sqrt{\frac{8 \mathrm{D} \alpha^{2}}{M}}|\sin (q a / 2)|, \quad|q| \leq \pi / \mathrm{a},
$$

where $a$ is the lattice constant, $\omega(\mathrm{q})$ and $q$ are the frequency and phonon wavenumber, and $M$ is the mass of composite atoms.

\section{Results and discussion}

To receive the interatomic Morse potential parameters, we need to calculate the parameter c in Eq. (10). The space lattice of the diamond is the fcc. The primordial basis has two identical atoms connected with each point of the fcc lattice, one atom at $\left(\begin{array}{ll}0 & 0\end{array}\right.$ $0)$ position, which has the atomic Wyckoff positions for the predicted phases at the ambient condition of $4 \mathrm{a}$, and one atom at (1/4 1/4 1/4) with the atomic Wyckoff positions of $8 \mathrm{c}$. Thus, the conventional unit cube contains eight atoms so that we obtain the value $c=1 / 4$ for this structure.

Applying the above derived expressions, we calculate thermal parameters for DIA structure crystals $(\mathrm{Si}, \mathrm{Ge}$, and $\mathrm{SiGe})$ using the lattice constants [11], the energy of sublimation [15], and the compressibility [20].

The numerical results of the interatomic Morse potential parameters are presented in Tables 1 and 3 . The theoretical values of $D, \alpha$ fit well with the experimental values [10, 14]. The elastic constants $c_{\mathrm{i}}$, effective spring force constants $k_{\text {eff }}$ and effective spring cubic parameters $k_{3 \text { eff }}$ calculated by interatomic Morse potential parameters for $\mathrm{Si}, \mathrm{Ge}$, and their alloys are presented in Tables 2 and 3 and compared to the experimental values $[11,15]$.

The calculated results for the state equation are illustrated in Fig. 2 for Si crystal and Fig. 3 for Ge crystal compared to the experimental ones (dashed line) [10] represented by an extrapolation procedure of the measured data. They show a good agreement between theoretical and experimental results, especially at low pressure.

Figures 4 and 5 illustrate good agreement of the anharmonic interatomic effective potentials for $\mathrm{Si}, \mathrm{Ge}$, and $\mathrm{SiGe}$ semiconductor calculated by using the present theory (solid line), and the experiment values obtained from interatomic Morse potential

Table 3 Morse potential parameters, spring force constants, and cubic parameters under pressure effects up to $14 \mathrm{GPa}$

\begin{tabular}{lllll}
\hline Pressure $(\mathrm{GPa})$ & $\boldsymbol{D}(\mathrm{eV})$ & $\boldsymbol{a}\left(\AA^{-\mathbf{1}}\right)$ & $\boldsymbol{k}_{\text {eff }}\left(\mathrm{eV} / \AA^{\mathbf{2}}\right)$ & $\boldsymbol{k}_{\text {3eff }}\left(\mathrm{eV} / \AA^{\mathbf{3}}\right)$ \\
\hline 0 & 0.3376 & 1.3588 & 3.1396 & 0.6423 \\
5 & 0.3154 & 1.3485 & 2.9032 & 0.6415 \\
10 & 0.2977 & 1.3168 & 2.7428 & 0.5902 \\
14 & 0.2184 & 1.2854 & 2.3595 & 0.5527 \\
\hline
\end{tabular}




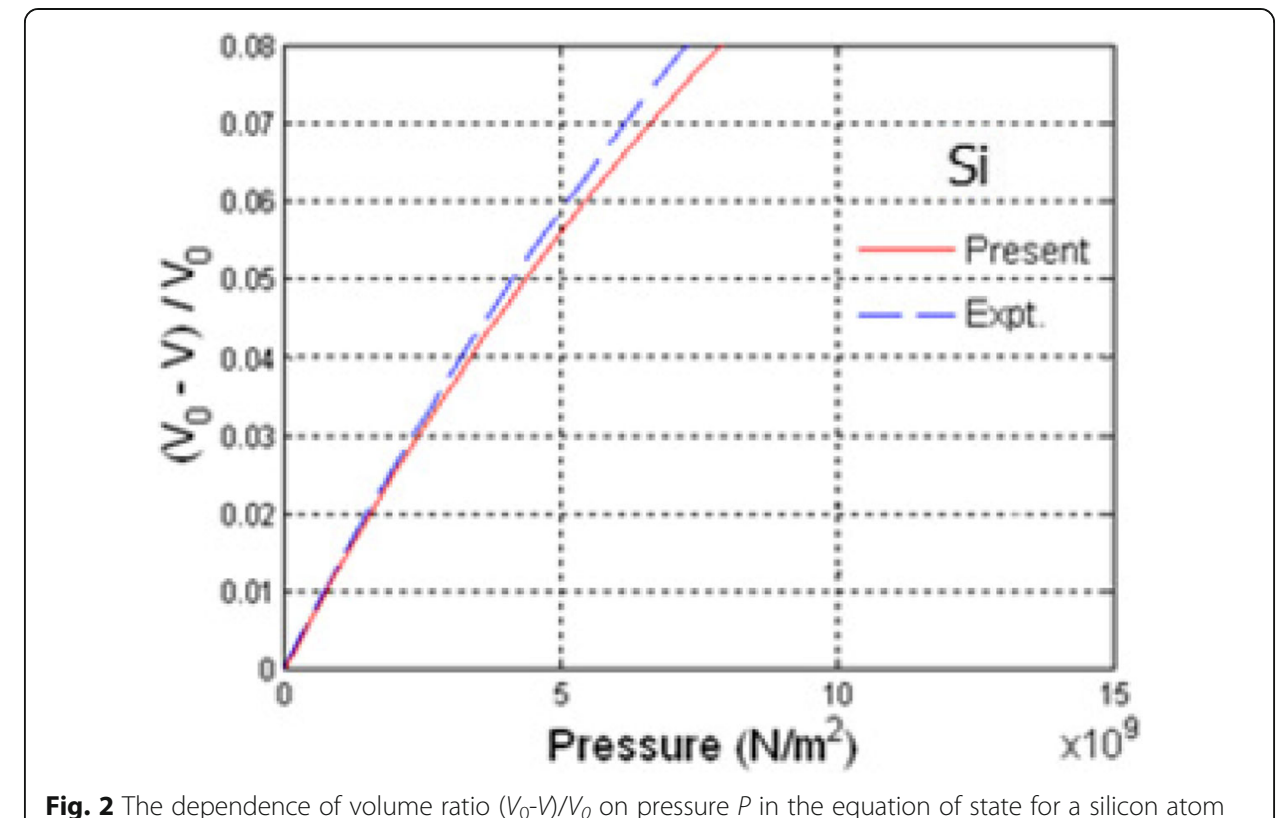

Fig. 2 The dependence of volume ratio $\left(V_{0}-V\right) / V_{0}$ on pressure $P$ in the equation of state for a silicon atom

parameters of J. C. Slater (solid line and symbol $\square$ ) [10], and simultaneously show strong asymmetry of these potentials due to the anharmonic contributions in atomic vibrations of these DIA structure crystals which are illustrated by their anharmonic shifting from the harmonic terms (dashed line).

Figures 6 and 7 shows dependence on pressure and temperature of $\operatorname{MSRD} \sigma^{2}(T)$ and MSD $u^{2}(T)$ for $\mathrm{Si}$ and Ge crystals. MSRD and MSD linear proportional to the temperature $T$ at high temperatures so the classical limit can be applied. At low temperatures, the curves of MSRD and MSRD for Si and Ge contain zero-point energy contributions; this is a quantum effect. The calculated results of MSRD and MSD for the $\mathrm{Si}, \mathrm{Ge}$ crystals agree well with the values of the experiment [10]. Thus, it is possible

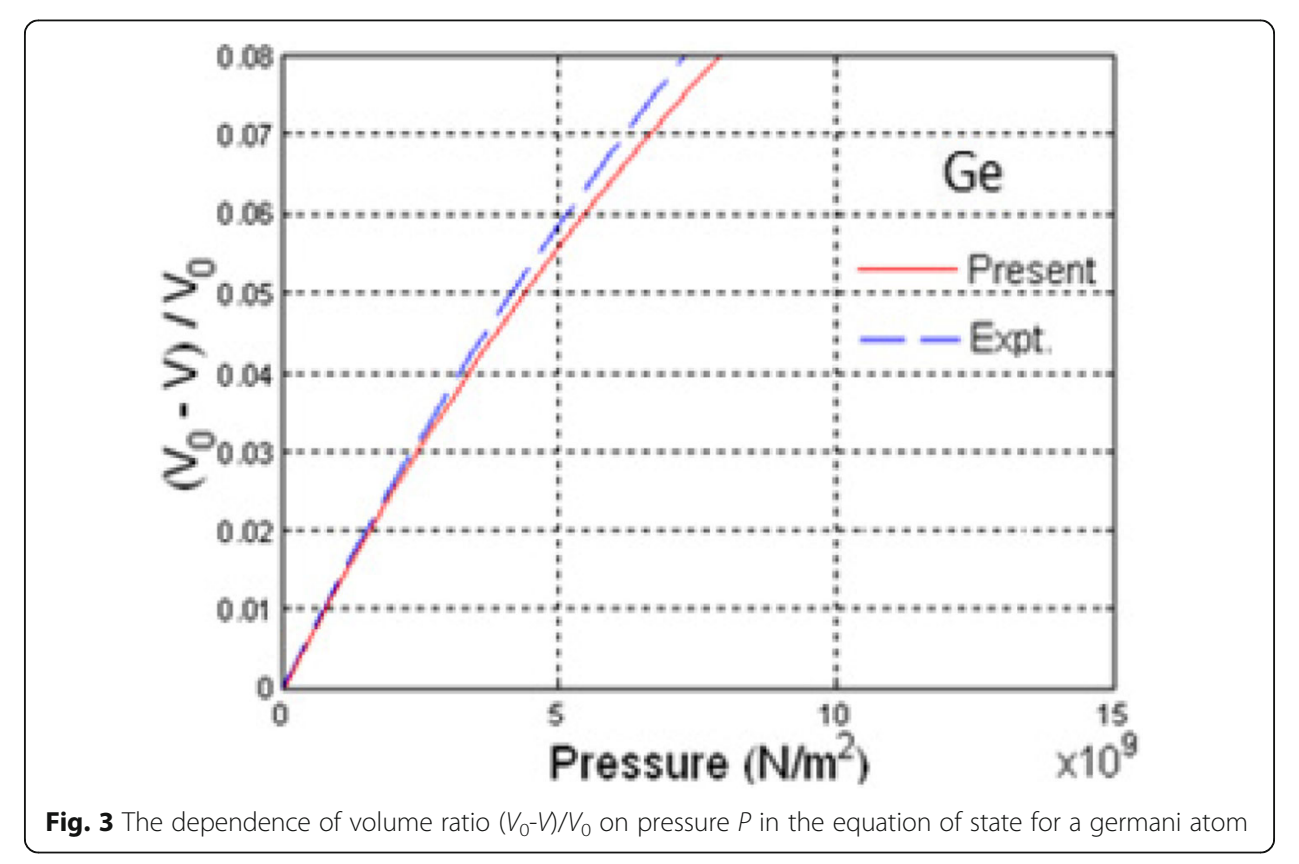




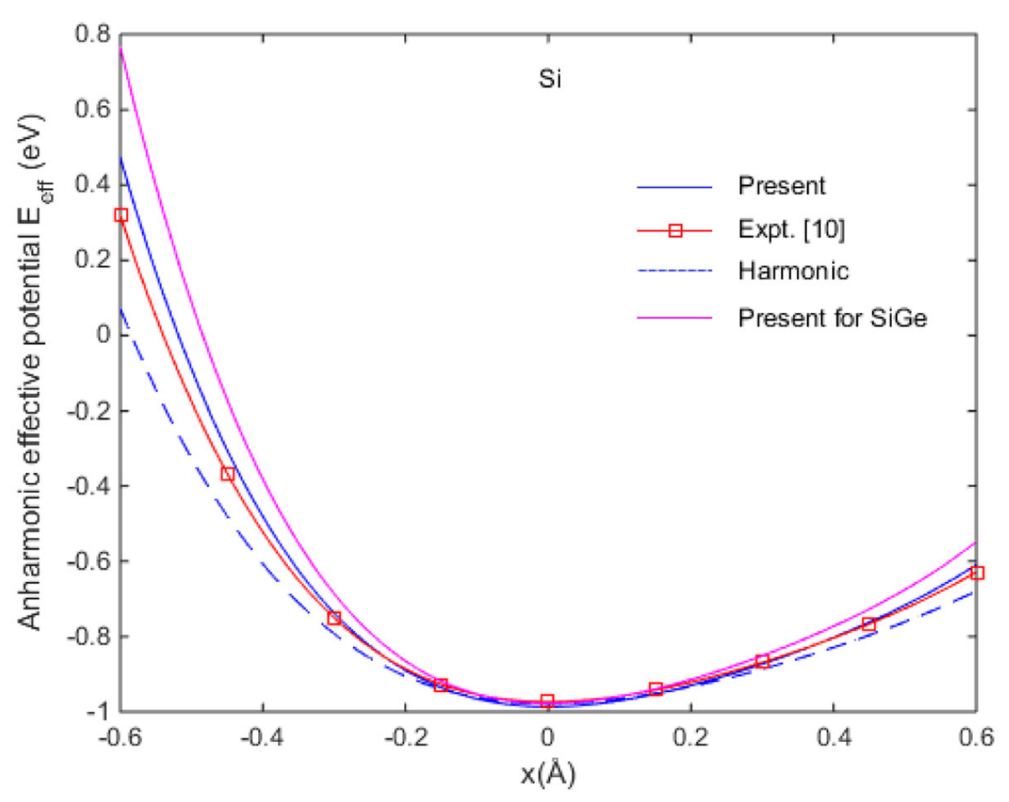

Fig. 4 Anharmonic effective potential for Si and SiGe semiconductor and comparison with harmonic effects

to deduce that the present proceduce for diamond-type structure crystals such as $\mathrm{Si}$, Ge crystals is reasonable.

\section{Conclusions}

In this work, a calculation method of interatomic Morse potential parameters and application for DIA and fcc structure crystals have been developed based on the calculation of volume and number of an atom in each basic cell and the sublimation energy,

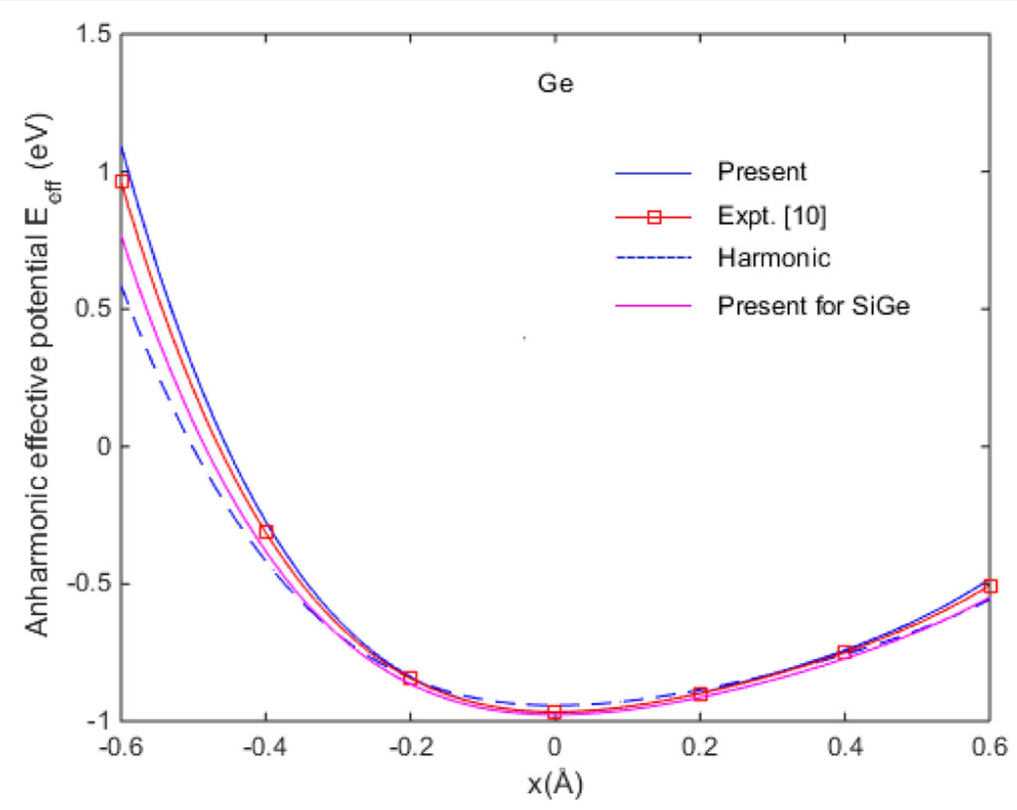

Fig. 5 Anharmonic effective potential for Ge and SiGe semiconductor and comparison with harmonic effects 


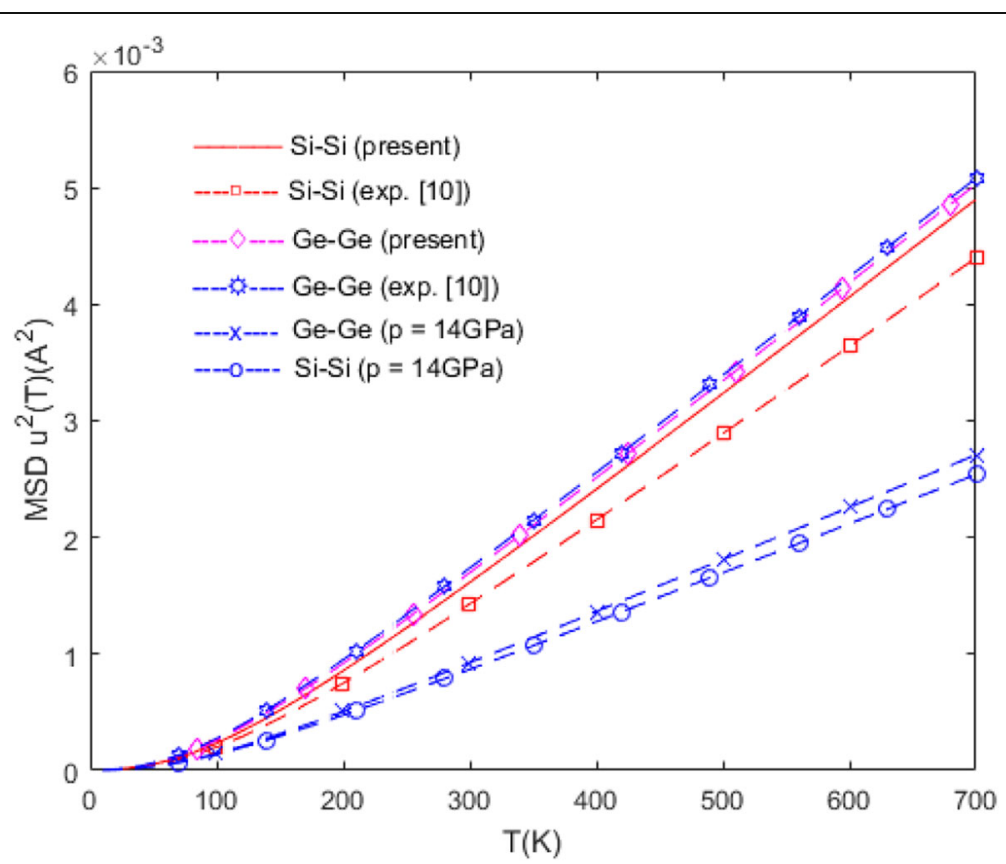

Fig. 6 Dependence on temperature of mean square displacement MSD under pressure effects up to $14 \mathrm{GPa}$

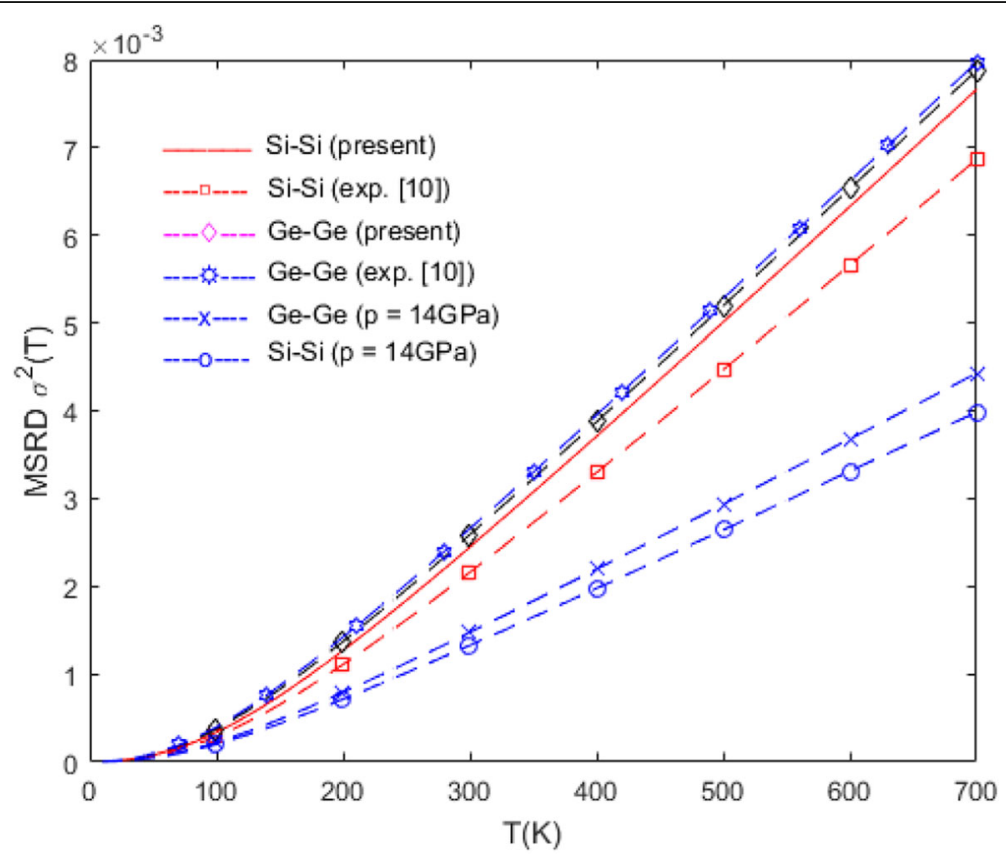

Fig. 7 Dependence on temperature of mean square relative displacement MSRD under pressure effects up to $14 \mathrm{GPa}$ 
compressibility, and lattice constant. The results have applied to the mean square relative displacement, mean square displacement, the state equation, the elastic constants, anharmonic interatomic effective potential, correlated function, and local force constant in EXAFS theory.

Derived equation of state and elastic constants satisfy all standard conditions for these values, for example, all elastic constants are positive. The interatomic Morse potentials obtained satisfy all their basic properties. They are reasonable for calculating and analyzing the anharmonic interatomic effective potentials describing anharmonic effects in EXAFS theory. This procedure can be generalized to the other crystal structures based on calculating their volume and number of an atom in each elementary cell.

Reasonable agreement between our calculated results and the experimental data show the efficiency of the present procedure. The calculation of potential atomic parameters is essential for estimating and analyzing physical effects in the EXAFS technique. It can solve the problems involving any deformation and of atom interaction in the diamond structure crystals.

\section{Abbreviations \\ EXAFS: Expanded X-ray Absorption Fine Structure; MSRD: Mean square relative displacement; MSD: Mean square displacement; DIA: Diamond-type; FCC: Face-centered cubic; DWF: Debye-Waller factor}

\section{Acknowledgements}

The author (DBN) thanks the Tan Trao University, Tuyen Quang, Vietnam, for support.

\section{Authors' contributions}

DBN (corresponding author) analyzed the structural data and conceptualized and wrote the manuscript. HPT collected the experimental data, and read, analyzed, and edited the errors in the manuscript. All authors have read and approved the manuscript.

\section{Authors' information}

- Professor Duc Ba Nguyen is a senior lecturer and researcher of Tan Trao University, Tuyen Quang, Vietnam. He had many studies that have been published in ISI, Scopus on the field of XAFS spectroscopy, and the material structure.

- Hiep Trinh Phi is a lecturer and researcher of Tan Trao University, Tuyen Quang, Vietnam. He studies XAFS spectroscopy and the material structure and has some published in the journal of science.

\section{Funding}

No funding was obtained for this study.

Availability of data and materials

All data generated or analyzed during this study are included in this published article.

\section{Declarations}

Competing interests

The authors declare that they have no competing interests.

Received: 12 July 2021 Accepted: 19 August 2021

Published online: 25 October 2021

\section{References}

1. Anzellini S, Monteseguro V, Bandiello E, Dewaele A, Burakovsky L, Errandonea D (2019) In situ characterization of the high pressure - high temperature melting curve of platinum. Sci Rep 9(1):13034. https://doi.org/10.1038/s41598-019-4 9676-y

2. Errandonea D, Burakovsky L, Preston DL, MacLeod SG, Santamaría-Perez D, Chen S, Cynn H, Simak SI, McMahon MI, Proctor JE, Mezouar M (2020) Experimental and theoretical confirmation of an orthorhombic phase transition in niobium at high pressure and temperature. Commun Mater 1(1):60. https://doi.org/10.1038/s43246-020-00058-2

3. Anzellini S, Burakovsky L, Turnbull R, Bandiello E, Errandonea D (2021) P-V-T equation of state of iridium up to $80 \mathrm{GPa}$ and 3100 K. Crysstal. 11(452). https://doi.org/10.3390/cryst11040452

4. Marques EC, Sandrom DR, Lytle FW, Greegor RB (1982) Determination of thermal amplitude of surface atoms in a supported Pt catalyst by EXAFS spectroscopy. J. Chem. Phys 77:1027. https://doi.org/10.1063/1.443914

5. Duc NB, Binh NT (2017) Statistical Physics-Theory and Application in XAFS, 173-198. Academic Publishing, LAP LAMBERT 
6. Hung NV, Duc NB, Frahm RR (2002) A new anharmonic factor and exafs including anharmonic contributions. J. Phys. Soc. Jpn. 72(5):1254-1259. https://doi.org/10.1143/JPSJ.72.1254

7. Frenkel Al, Rehr JJ (1993) Thermal expansion and x-ray-absorption fine-structure cumulants. Phys. Rev. B 48:585. https:// doi.org/10.1103/PhysRevB.48.585

8. Girifalco LA, Weizer VG (1959) Application of the Morse potential function to cubic metals. Phys. Rev. 114(3):687-690. https://doi.org/10.1103/PhysRev.114.687

9. Hung NV, Rehr JJ (1997) Anharmonic correlated Einstein-model Debye-Waller factors. Phys. Rev. B 56(1):43-46. https:/ doi.org/10.1103/PhysRevB.56.43

10. Pirog IV, Nedoseikina TI, Zarubin Al, Shuvaev AT (2002) Anharmonic pair potential study in face-centred-cubic structure metals. J. Phys.: Condens. Matter 14(8):1825-1832. https://doi.org/10.1088/0953-8984/14/8/311

11. Sydney P, Clark J (1996) Handbook of Physical Constants Geological Society of America

12. Okube M, Yoshiasa A (2001) Anharmonic effective pair potentials of group VIII and Lb Fcc metals. J. Synchrotron Radiat. 8(2):937-939. https://doi.org/10.1107/s0909049500021051

13. Miyanaga T, Fujikawa T (1994) Quantum statistical approach to Debye-Waller Factor in EXAFS, EELS and ARXPS. III. Applicability of Debye and Einstein Approximation. J. Phys. Soc. Jpn 63(1036):3683-3690. https://doi.org/10.1143/JPSJ. 63.3683

14. Greegor RB, Lytle FW (1979) Extended $x$-ray absorption fine structure determination of thermal disorder in Cu: comparison of theory and experiment. Phys. Rev. B 20(12):4908-4907. https://doi.org/10.1103/PhysRevB.20.4902

15. Slater, J. C. 1939). Introduction to Chemical Physics, (ed. McGraw) (Hill Book Company, Inc., New York,

16. Charles Kittel, 1986) Introduction to Solid-State Physics, (John Wiley \& Sons ed.), (Inc. New York,

17. Born M, Huang K (1956) Dynamical Theory of Crystal Lattice, 2nd edn. Clarendon Press, Oxford

18. Errandonea D, Ferrer-Roca C, Martínez-Garcia D, Segura A, Gomis O, Muñoz A, Rodríguez-Hernández P, López-Solano J, Alconchel S, Sapiña F (2010) High-pressure x-ray diffraction and ab initio study of NizMo3N, Pd2Mo3N, Pt2Mo3N, Co3Mo3N, and Fe3Mo3N: two families of ultra-incompressible bimetallic interstitial nitrides. Phys. Rev. B 82(17):174105. https://doi.org/10.1103/PhysRevB.82.174105

19. Duc NB (2020) Influence of temperature and pressure on cumulants and thermodynamic parameters of intermetallic alloy based on anharmonic correlated Einstein model in EXAFS. In: Application of the Debye model to study anharmonic correlation effects for the CuAgX ( $X=72 ; 50)$ intermetallic alloy, Physica Scripta, 95. https://doi.org/10.1 088/1402-4896/ab90bf

20. Bridgeman, P. W. (1940) The Compression of 46 Substances to $50,000 \mathrm{~kg} / \mathrm{cm}$. Proceedings of the American Academy of Arts and Sciences, 74, 3, 21-51. DOl: 10.2307/20023352

\section{Publisher's Note}

Springer Nature remains neutral with regard to jurisdictional claims in published maps and institutional affiliations.

\section{Submit your manuscript to a SpringerOpen ${ }^{\circ}$ journal and benefit from:}

- Convenient online submission

- Rigorous peer review

- Open access: articles freely available online

High visibility within the field

- Retaining the copyright to your article

Submit your next manuscript at $\boldsymbol{\nabla}$ springeropen.com 